\title{
A Computational Model of Non-optimal Suspiciousness in the Minnesota Trust Game
}

\author{
Rebecca Kazinka ${ }^{1}$, Iris Vilares ${ }^{2}$, Angus W. MacDonald III
}

1. Graduate Program in Clinical Science and Psychopathology Research, University of Minnesota, Minneapolis, MN, United States of America

2. Psychology Department, University of Minnesota, Minneapolis, MN, United States of America

Corresponding author: angus@umn.edu

Authors Note: This is a preprint of our original research and a draft of our manuscript as of $08 / 17 / 2021$. It has not yet been peer-reviewed. Funding for data collection was provided by the University of Minnesota. This project was supported from U.S. National Institutes of Mental Health grants T32-MH115688 (RK) and R21MH112918 (AM). 


\title{
MODELING PERSECUTORY IDEATION
}

\begin{abstract}
This study modelled spite sensitivity (the worry that others are willing to incur a loss to hurt you), which is thought to undergird suspiciousness and persecutory ideation. Two samples performed a parametric, non-iterative trust game known as the Minnesota Trust Game (MTG). The MTG is designed to distinguish suspicious decision-making from otherwise rational mistrust by incentivizing the player to trust in certain situations. Individuals who do not trust even under these circumstances are particularly suspicious of their potential partner's intentions. In Sample 1, 243 undergraduates who completed the MTG showed less trust as the amount of money they could lose increased. However, for choices where partners had a financial disincentive to betray the player, variation in the willingness to trust the partner was associated with suspicious beliefs. To further examine spite sensitivity, we modified the Fehr-Schmidt (1999) inequity aversion model, which compares unequal outcomes in social decision-making tasks, to include the possibility for spite sensitivity. In this case, an anticipated partner's dislike of advantageous inequity (i.e., guilt) parameter could take on negative values, with negative guilt indicating spite. We hypothesized that the anticipated guilt parameter would be strongly related to suspicious beliefs. Our modification of the Fehr-Schmidt model improved estimation of MTG behavior. We isolated the estimation of partner's spite-guilt, which was highly correlated with choices most associated with persecutory ideation. We replicated our findings in a second sample, where the estimated spite-guilt parameter correlated with self-reported suspiciousness. The "Suspiciousness" condition, unique to the MTG, can be modeled to isolate
\end{abstract}




\section{MODELING PERSECUTORY IDEATION}

spite sensitivity, suggesting that spite sensitivity is separate from inequity aversion or risk aversion, and may provide a means to quantify persecution. The MTG offers promise for future studies to quantify persecutory beliefs in clinical populations.

Keywords: Spite sensitivity, trust, suspiciousness, decision-making, risk aversion, computational modeling, inequity aversion 
MODELING PERSECUTORY IDEATION

\section{Author Summary}

Our study examined persecutory ideation in an undergraduate sample using an economic game played between two anonymous players. Participants in the study had to choose whether or not to allow a partner to decide between fair and unfair distributions of money. Most importantly, in some cases the partner was offered less money if they chose the unfair option than if they chose the fair option, such that the main incentive for the partner to select the unfair option would be to hurt the participant. We posited that selecting this unfair option represented a partner's spitefulness; therefore, participants who did not trust during this condition were likely motivated by a fear of spite. We created a mathematical model to measure this fear of spite in the participant to replicate observed average behavior in the Minnesota Trust Game, as well as to compare it to personality measures related to fears of persecution. We found that our measurement of fear of spite was highly correlated with behavior in the suspicious choices. This mathematical model is a first step to quantify persecutory ideation in this economic game, and it could in the future be used in clinical populations where these beliefs are common and distressing. 


\section{MODELING PERSECUTORY IDEATION}

\section{Introduction}

Social interactions can be sorted broadly into 4 categories -- cooperative, selfish, altruistic, and spiteful -- based on the consequences (positive or negative) for the actor and the recipient (1). Cooperative mutual benefits occur when both individuals have positive outcomes. When an individual chooses to gain when another must take a loss it is selfish. In contrast, altruism occurs when the actor takes a loss so that others can gain. These social interactions have received a good deal of attention in recent decades. The final category is less commonly studied: spite occurs when the actor is willing to take a loss to ensure that a partner also loses. Initially, spite may seem irrational because it leads to a loss for an individual. However, spite may have evolved as a form of kin selection, in which spite toward an unrelated recipient may endure when the beneficiary is genetically related, in a similar manner as altruism $(1,2)$. An example in nature can be seen in the sterile soldier caste in poly-embryonic parasitoid wasps. The wasp eggs divide asexually when they are laid on the eggs of moth caterpillars, and small portion become the sterile soldier caste. To create the soldier caste is costly to the wasp, as it takes energy to create new life, yet they will not reproduce. Additionally, the soldier caste is costly to other wasp larvae because soldiers preferentially seek out and kill larvae that are less related to themselves (3). While this may first seem counterintuitive, this behavior frees up resources for their clone-mates, thus improving the chances of their kin surviving. For more examples in nature, see review by West \& Gardner (2). Spite, therefore, may provide useful insights into a drive towards seemingly irrational negative outcomes in social interactions. 


\section{MODELING PERSECUTORY IDEATION}

The existence of spite may provide clues for our understanding of a fear of spiteful partners, which we propose represents a fear of persecution. A fear of persecution, or increased suspiciousness that others are out to get oneself, is characteristic of psychotic disorders like schizophrenia (4), but also exists within approximately $11 \%$ of non-clinical samples in the general population (5-7). One possible mechanism for non-psychiatric suspiciousness of others may be this fear of their spiteful behavior. If an individual is socially alienated, they may be more inclined to interpret others' behavior as spiteful. Therefore, strong priors that partners will be spiteful may provide a mechanism for persecution. Past research has suggested that increased self-reported suspiciousness, or alienation, is associated with spite sensitivity, or an individual's fear that others are willing to incur a cost to themselves to cause the greater harm to the participant (8). This finding supports the framework that a fear of spite in social interactions may be key to understanding the fear of persecution seen both in the general population and in more serious clinical populations.

One useful way to approach an individual's beliefs and motivations in a social interaction is through the use of game theory and social decision-making games. Game theory can be a valuable way to measure intentions through actions instead of through interview or questionnaire, which can be affected by malingering, faking good, or lack of insight (9). Social decision-making games can therefore be used to measure beliefs through choices impacted by consequences, in which we can assess beliefs about outcomes rather than relying on selfreport. Several social decision-making games exist (see reviews 10-14) where players make decisions that have consequences for both themselves and others. In these games, players 


\section{MODELING PERSECUTORY IDEATION}

decide how to interact with their partner based on intuitions about how their partner will respond, allowing for estimation of their prior beliefs. Such social decision-making games have distilled complex constructs into straightforward interactions to assess participants' beliefs.

One such social decision-making game is the Trust Game (15), in which two players make decisions in succession about whether to cooperate or betray their partner. Player 1 (the investor) has the option to split an amount of money (e.g.: \$10) and give some portion (or all) to their partner player 2; it is then multiplied (usually by 3 ), given to player 2 (the trustee), and then player 2 can decide how much to return. The amount given by the investor is used as a proxy for the level of trust they have in the trustee, and the amount returned by the trustee is used to measure their level of trustworthiness. Individuals with psychosis, who commonly experience delusions of persecution (16), have been shown to trust less when playing as the investor $(17,18)$. Thus, the Trust Game provides a useful foundation for understanding suspiciousness of partners, yet it is difficult to disentangle beliefs about the partner as suspiciousness, as rational mistrust of a predictably selfish partner, or as an aversion to uncertainty more generally.

In the typical Trust Game, it is reasonable that people are concerned that their partners are untrustworthy, given the competing gains for player 1 and player 2 , and therefore players may be less willing to trust the partner. But what if player 2 incurs a cost in order for player 1 to lose money? In this instance, player 2 would be acting spitefully, and thus a non-optimal suspiciousness toward player 2 may indicate sensitivity to spite. As illustrated in Figure 1, The Minnesota Trust Game (MTG) incorporates two conditions with outcomes dichotomized into 


\section{MODELING PERSECUTORY IDEATION}

two choices for each player. We refer to players based on the order of decision making, i.e., the first mover and second mover. The first mover (player 1) can choose to take a small, safe outcome $(S)$ and end the game (no trust) or trust the second mover (player 2 ) to choose between two possible divisions of money. In one condition, the Rational Mistrust condition, the second mover can either choose a fair, mutually beneficial outcome $(M)$, much larger than $S$, or an outcome that yields a temptingly higher payoff $(T)$ for the second mover and adverse payoff (Ad) for first mover. As Ad decreases in value - even including negative values - the first mover's shift from trusting the second mover to not trusting them and choosing the safe outcome reveals their level of rational mistrust in this condition. In a second condition, the Suspiciousness condition, the second mover is instead offered a temptation $(T)$ that is less than the fair option, such that it is monetarily advantageous for the second mover to choose the fair option $M$. Therefore, it is reasonable to trust the second mover because they have an incentive to cooperate and would incur a monetary cost to hurt the first mover; the first mover may not trust in this condition if they have increased suspiciousness of the second mover (Figure 1). Participants also play this game against a fair coin to examine differences in trust depending on if the partner is another human or indifferent chance. This additional comparison disentangles the first mover's beliefs about intention and risk aversion, thus the conditions against the coin are referred to as Risk Aversion. The added dimension of the Suspiciousness condition allows us to measure spite sensitivity, unlike the original Trust Game. 
a.
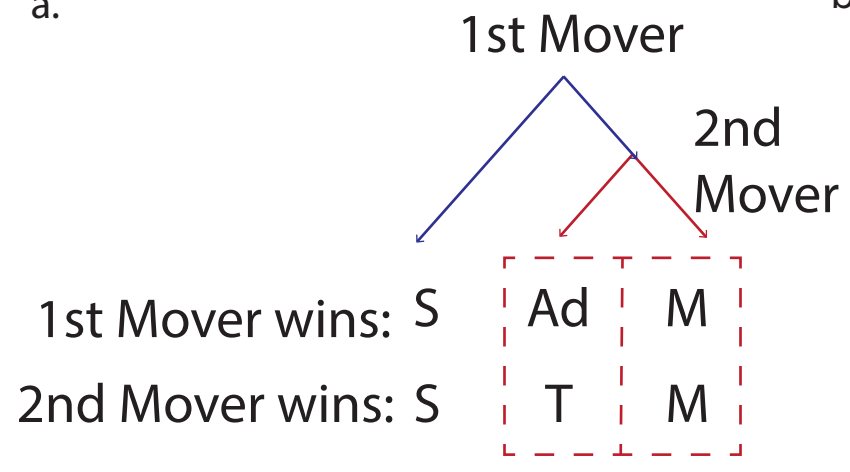

\begin{tabular}{|c|c|c|c|c|}
\hline & S & M & \multicolumn{1}{|c|}{ T } & \multicolumn{1}{c|}{ Ad } \\
\hline Sample 1 & 10 & 20 & $\begin{array}{l}25 \text { (RMT) } \\
15 \text { (SUS) }\end{array}$ & $\begin{array}{l}-5 \text { to } 15 \\
\text { by } 1 \text { 's }\end{array}$ \\
\hline Sample 2 & 8 & 12 & $\begin{array}{l}14 \text { (RMT) } \\
10 \text { (SUS) }\end{array}$ & $\begin{array}{l}-5,0,3,5, \\
6,7,8\end{array}$ \\
\hline
\end{tabular}

b.

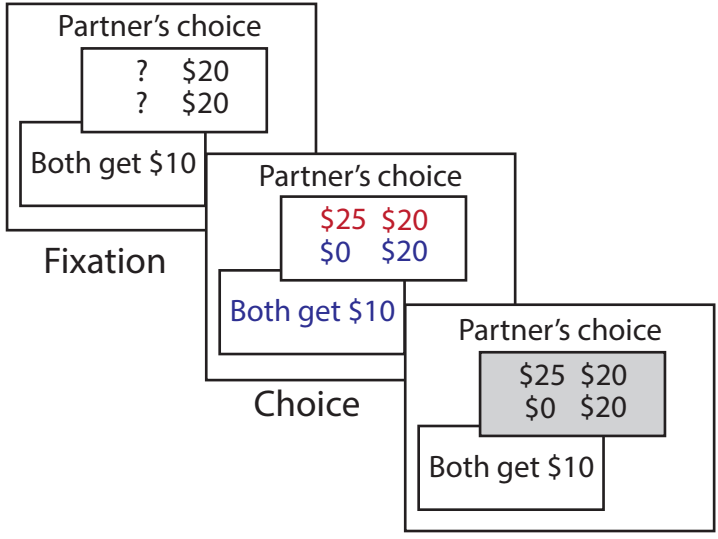

Feedback

Figure 1. Diagram of gameplay. a. The Decision Tree of the Minnesota Trust Game shows possible outcomes for each player. The first mover first chooses between both players getting a small reward (S) or letting the second mover decide. The second mover can choose between both players getting a larger mutual reward (M) or an adverse payoff for the first mover (Ad) and a temptation $T$ for the second mover. The table describes the values for $S, M, T$, and Ad for the two samples. The Suspiciousness condition is referred to as 'SUS' and the Rational Mistrust condition is referred to as 'RMT' when playing against a human partner. Participants also play against a fair coin, which are referred to as Risk aversion conditions or ' $R A(T)$ ', depending on the $T$ amount. $\boldsymbol{b}$. An example trial of the Rational Mistrust condition in the First Mover Game, with $T$ $=25$ and $A d=0$ (Sample 1). Participants saw question marks for the variable outcomes during the fixation, and then had a fixed amount of time to choose when the values were displayed. Their choice was confirmed during the feedback window; however, they never received feedback about the choice made by the second mover.

Findings from the Minnesota Trust Game from our lab showed that individuals were more trusting in the Suspiciousness condition compare to the Rational Mistrust condition when playing against a human partner versus a random coin (8). However, there was variability in the amount that individuals were willing to trust in the Suspiciousness condition. Individuals who 


\section{MODELING PERSECUTORY IDEATION}

reported higher Alienation, a Multiphasic Personality Questionnaire (MPQ) (19) subscale measuring beliefs like betrayal and isolation, were less trusting in the Suspiciousness condition. Additionally, this game showed differences in the Risk Aversion (random coin partner) responses when comparing high and low Harm Avoidance, a MPQ subscale measuring risk aversion (8). These results show evidence of individual differences in the MTG that could therefore be more precisely estimated using a computational model to measure spite sensitivity in this two-player game.

One approach to understanding the interaction of players in social decision-making games is to create a normative model of behavior to explain decision-making processes. Fehr \& Schmidt (1999) first proposed a normative model of behavior in several social decision-making games that involved splitting money between individuals (20). This inequity aversion model uses two parameters to calculate utility of the options: a player's guilt ( $\beta$; dislike of advantageous inequality) and envy ( $\alpha$; dislike of disadvantageous inequality) by calculating the modifier of the difference between the outcomes for each player (Eq. 1). From the utility of options, this model can be used to describe behavior when an individual is maximizing the utility of an outcome, incorporating social utilities (based on parameters) along with monetary value. Importantly, this model adds a deeper understanding of individual differences, rather than simply relying on aggregate analysis of the game such as the Nash equilibrium $(21,22)$. The Fehr-Schmidt model therefore establishes a simple explanation for choices to cooperate with a partner based on the dislike of inequity between the two players. 


\section{MODELING PERSECUTORY IDEATION}

The Fehr-Schmidt model has been widely adapted for different scenarios and social decision-making games (23-26). The Equity, Reciprocity, and Competition (ERC) model (27) builds off of the Fehr-Schmidt model and focuses on these three features of social interactions by comparing the relative payoffs between players. It assumes that the motivation of the player includes both the player's monetary payoff as well as the relative share of the payoff that they are receiving. For example, in the Ultimatum Game, where a responder decides whether or not to accept a division of money from a proposer, a responder's need for equity drives the outcome, such that proposers respond more fairly to keep the relative share of the payoff higher (27). Another example includes the modification of the Fehr-Schmidt model to examine a multi-round trust game (26). This model integrated with an interactive partially observable Markov decision process (I-POMDP) and added parameters for theory of mind, planning, risk, irritability, and irritability belief to explain behavior of trust, reciprocity, and betrayal when individuals learned to respond over several rounds playing with the same individual. This model specifically calculates theory of mind, i.e. the understanding of another's intentions or beliefs (28), by assessing the level of interplay in responses over time. These adapted models reveal more information about complex beliefs of an individual when making decisions building on the Fehr-Schmidt model. They show that adaption of this model can provide insight on subtle aspects of social decision-making. However, the Fehr-Schmidt model does not model outcomes in the MTG, and to date we have not identified another model that assesses spite sensitivity, thus leaving a gap in understanding about this area of social interaction. Therefore, we aim to 


\section{MODELING PERSECUTORY IDEATION}

adapt the Fehr-Schmidt model to examine an important but understudied phenomenon, spite sensitivity, using the Minnesota Trust Game (8).

\section{The current study}

In this study we developed a computational model of Minnesota Trust Game first mover game ( $1^{\text {st }} \mathrm{M}$ Game), with the aim of replicating observed MTG behavioral results and more accurately measuring spite sensitivity. Our model is adapted from the Fehr-Schmidt inequity aversion model (20), which parameterizes play between two individuals who may receive unequal rewards. The Fehr-Schmidt model does not explain instances when individuals do not trust when it might be logical, from a monetary perspective, to assume the second mover will be trustworthy. Therefore, we tested several parameters to create the best fitting model for the MTG in two samples, one for model development and a second for replication and extension of our primary hypotheses. Below are the hypotheses we tested for our model, which were developed before testing.

H1. The Fehr-Schmidt model will outperform a random model, which assumes that an individual would have a 50\% chance of choosing either option (go for the safe option or trust). This test was a validity check to ensure that the Fehr-Schmidt model was an appropriate base model guide in our modeling.

H2. Behavior will differ between decision agents (the coin and human partner) and will therefore require separate parameters to explain participants' efforts to account for their partners' incentives. Due to the difference in behavior between the two decision agents, we tested model fits that applied additional parameters for the human decision agent.

H3. Risk aversion $\left(R_{i}\right)$ will partially but not fully explain the distinction between decisionagent conditions (comparing coin and human partner). Risk aversion was tested by 


\section{MODELING PERSECUTORY IDEATION}

modifying the value of the difference between the first mover's potential outcome against the safe assured payoff $(S)$. We hypothesized a risk aversion parameter would be necessary for the model to explain overall risk aversion. However, risk aversion alone would not distinguish the behavioral differences seen between the Rational Mistrust and Suspiciousness conditions.

In addition to testing these hypotheses, we compared two different models to assess spite sensitivity by modeling an estimation of the partner's choice:

H4a. Model spite sensitivity by allowing the estimated second mover's guilt to be negative. We applied the Fehr-Schmidt model to the partner's choice to measure the first mover's estimation of the second mover's guilt for getting more money. Importantly, we allowed the second mover's guilt to become negative, which was not the case in the original Fehr-Schmidt model. Fehr \& Schmidt reported that they constrained guilt to be positive to "rule out the existence of subjects who like to be better off than others" (p. 824, Fehr \& Schmidt, 1999); they posited that while these people likely exist, in the context of the experiment it would have minimal impact on equilibrium behavior. Our reasoning for including negative values for guilt is that if positive second mover inferred guilt means that the first mover believes their partner would be fairer, then negative second mover guilt would mean that the first mover believes the partner would be spiteful (and actually be willing to incur a cost to inflict a higher cost on their partner). While in the original Trust Game, one would not anticipate that negative values would be common or relevant to the question at hand; in the MTG we hypothesized that negative guilt would provide insight into sensitivity to spite specifically, as it is expected to represent those who believe the second mover gets the most value out of being better off than the first mover. We hypothesized that the estimated spite-guilt parameter, where positive values represent guilt and negative values represent spite, would therefore be most correlated with the Suspiciousness condition, where the utility of inequity for the second mover (i.e., earning more than the first mover) is pitted directly against earning more money.

H4b. Model spite sensitivity separate from the estimated second mover's guilt, such that guilt and spite are both non-negative parameters. Like hypothesis 4a, we tested whether guilt and spite were separate measures, as compared to negative or positive guilt. Here, we created two parameters both constrained to be positive, and set the 


\section{MODELING PERSECUTORY IDEATION}

analysis so that when modeling the second mover's value of their choices, guilt reduced the utility of the choice of having more than the first mover while spite increased its utility.

\section{Results}

Our goal was to replicate behavioral results in the MTG and develop a model of spite sensitivity using the game. We also explored individual differences comparing personality measures and parameter estimates from the model. We developed and compared several models using Sample 1 of 243 undergraduate students. Once the model was established, we replicated results in Sample 2, consisting of 73 undergraduate students from a previously published sample (8). See Figure 1 and methods for more details about the differences between the samples and games.

\section{Behavioral results}

Looking at the behavior in the $1^{\text {st }} \mathrm{M}$ Game, we found that participants from Sample 1 showed similar behavior in the Risk Aversion conditions, yet less trust in the Rational Mistrust (\$25) condition and greater trust in the Suspiciousness (\$15) condition, although still well below $100 \%$. A repeated measures logistic regression (temptation $\mathrm{x}$ decision agent $\mathrm{x}$ adverse payoff) identified a three-way interaction $(\beta=.002, \mathrm{SE}=.0002, t=10.2, p<.001)$. Figure 2a shows the interaction of temptation $\mathrm{x}$ decision agent $(\beta=-.037, \mathrm{SE}=.001, t=-27.8, p<.001)$, in which there were differences in trust between the human partner conditions, but not coin conditions. This result confirms that participants treated the conditions for the human partners differently 


\section{MODELING PERSECUTORY IDEATION}

than the conditions with coin partners. Additionally, there were the predicted interactions of temptation $\mathrm{x}$ adverse payoff $(\beta=-.0004, \mathrm{SE}=.00012, t=-3.63, p<.001)$ and adverse payoff $\mathrm{x}$ decision agent $(\beta=-.051, \mathrm{SE}=.004, t=-14.7, p<.001)$. The relationship between temptation and adverse payoff showed that participants were more likely to trust in high-risk trials during the Suspiciousness condition compared to the Rational Mistrust condition. The adverse payoff $x$ decision agent interaction showed that overall, participants were more trusting with the human conditions for lower values compared to the coin condition. We found main effects of temptation, decision agent, and adverse payoff ( $\left.p^{\prime} s<.01\right)$. Overall, these results replicated past findings in the Minnesota Trust Game, such that participants were more trusting in the Suspiciousness condition than the Rational Mistrust condition, but there was no difference between the two coin conditions (8). 


\section{MODELING PERSECUTORY IDEATION}

a.

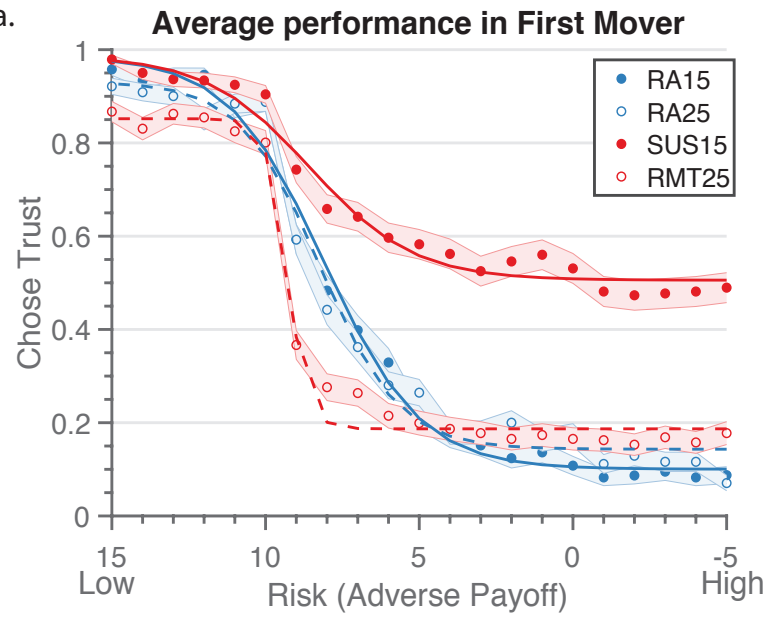

c.

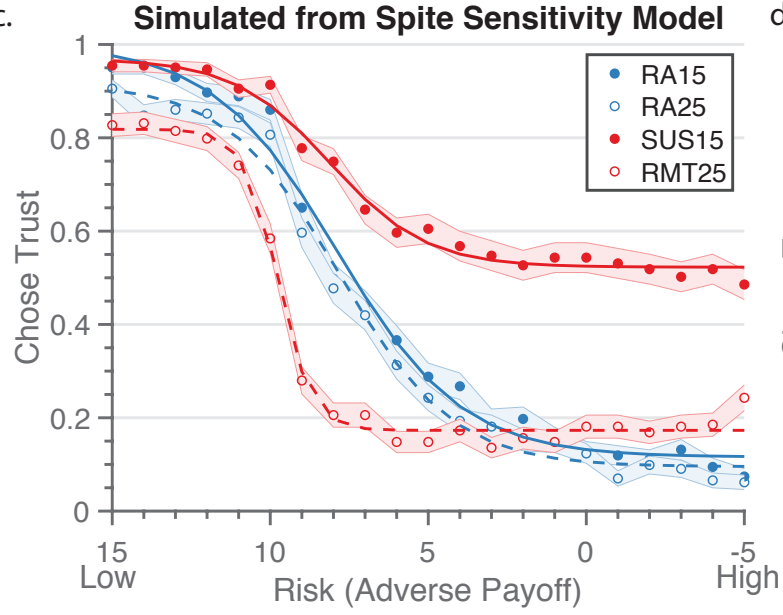

b.

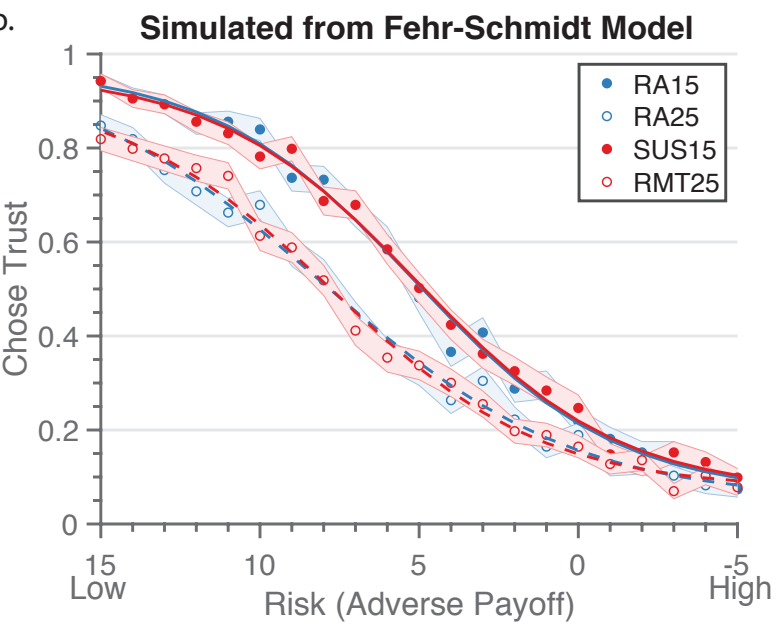

d. Recovered Simulation from Spite Sensitivity Model

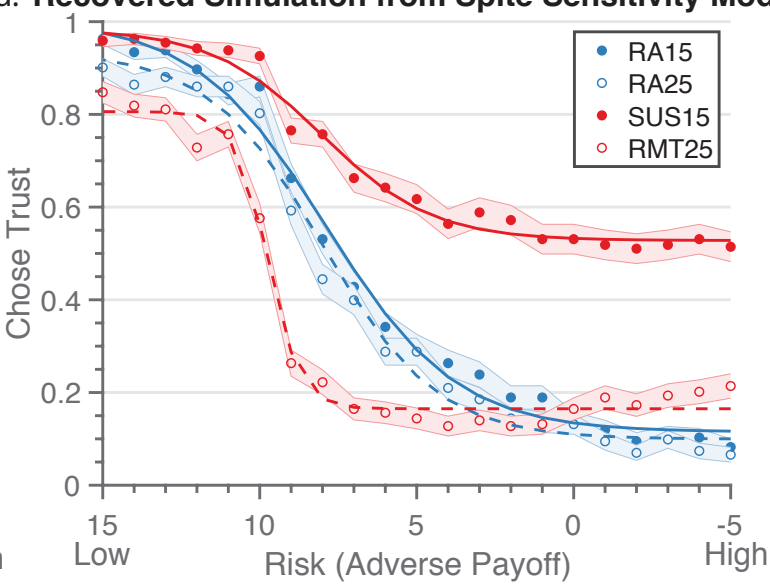

Figure 2. Behavior and Model Simulations. a) Original Sample 1 aggregate behavior. b) Simulated data from the original Fehr-Schmidt model, which does not match the pattern of Sample 1 aggregate behavior well. c) Simulated data from the best-fitting Spite Sensitivity model. d) We recovered parameters based on simulated data created in $2 c$ and then resimulated data based on the recovered parameters to test the stability of the model. Behavior is very similar to that seen in Figures $2 a$ and $2 c$. Note, risk increases along the $X$-axis, as indicated by the decreasing Adverse Payoff. RA15 is the low temptation condition against the coin. RA25 is the high temptation condition with the coin. SUS15 is the Suspiciousness condition with the human partner. RMT25 is the Rational Mistrust condition with the human partner. The shading indicates $95 \% \mathrm{Cl}$. 


\section{MODELING PERSECUTORY IDEATION}

\section{Model fitting}

Our best-fitting model focused on behavioral realism to explain the spite sensitivity phenomenon using several parameters. Importantly, we set our goals to identify a model that had both a good fit (using Bayesian Criterion Information) and replication of the expected pattern of behavior seen in prior work, in which there was a significant increase in trust in the Suspiciousness condition only for the human partner. We estimated the parameters for each individual and then simulated behavioral data using those parameters to test for replication of the actual behavior within the sample. We used the Fehr-Schmidt inequity aversion parameter envy $(\alpha)$ for the first mover, and additionally set up the model to measure the first mover's estimation of the second mover's guilt $\left(\beta^{\prime}\right)$. Our model extends into negative values, where positive values represent a dislike of advantageous inequity (guilt), zero represents indifference, and negative values represent an enjoyment of advantageous inequity (spite). We refer to this parameter as estimated spite-guilt to represent the continuum. In addition, we included risk aversion parameters, which modulated the difference between the safe amount of money and the potential adverse payoff. One risk aversion parameter was set for all trials, while a second risk aversion parameter was added for only the human partner (which we refer to as social risk aversion). Finally, a softmax equation was used to estimate the inverse temperature (i.e., noisiness) of decision making of the player, represented by lambda ( $\lambda$ ). See methods section for more details. 


\section{MODELING PERSECUTORY IDEATION}

\section{Model comparisons}

To test models, we compared both BIC values and accuracy of simulated data in describing behavior (Table S1). The Fehr-Schmidt model (which included parameters for envy and inverse temperature) improved upon a random model (supplemental figure S1); however, it does not separate out the interaction between condition and decision agent (Figure 2b). Our best-fitting model relied on three additional parameters to do this: two risk aversion parameters (general risk aversion, applied to all conditions, and social risk aversion, additionally applied only to the human partner conditions) and estimated spite-guilt. The best-fitting model, referred to as the Spite Sensitivity Model, successfully reproduced the interaction of condition and decision agent; additional recovery of the model suggests good reproducibility (Figure 2c \& 2d). Additional model comparisons tested risk aversion, estimated spite-guilt, separation of parameters across the decision agents, and spite as a separate parameter from guilt. Descriptions of model testing and results can be found in the supplemental materials. Importantly, estimated spite-guilt as a single continuous parameter performed better than separating estimated spite and estimated guilt, suggesting that $\mathrm{H} 4 \mathrm{a}$ is better than $\mathrm{H} 4 \mathrm{~b}$.

To test the replicability of the fit, we applied our best-fitting model (the Spite Sensitivity model) to Sample 2, 73 participants from Johnson et al. (2009)(8). The pattern of behavior in Sample 2 matched findings in Sample 1, in which the two coin conditions were very similar, yet participants were less trusting in the Rational Mistrust condition, but more trusting in the Suspiciousness condition (Figure 3a). Specific behavioral results from this dataset can be found 


\section{MODELING PERSECUTORY IDEATION}

in Johnson et al. (2009) (8). The simulated data from the Spite Sensitivity Model show the distinctions seen in the human partner between the Rational Mistrust and Suspiciousness conditions, as well as the little difference between the two Risk Aversion conditions. However, the simulated data of the Rational Mistrust condition underestimated the trust levels observed in the higher adverse payoff ranges (Figure 3b). Overall, the model adequately replicated the original behavior of the task, thus meeting our goal of generally fitting the pattern behavior seen in participants.
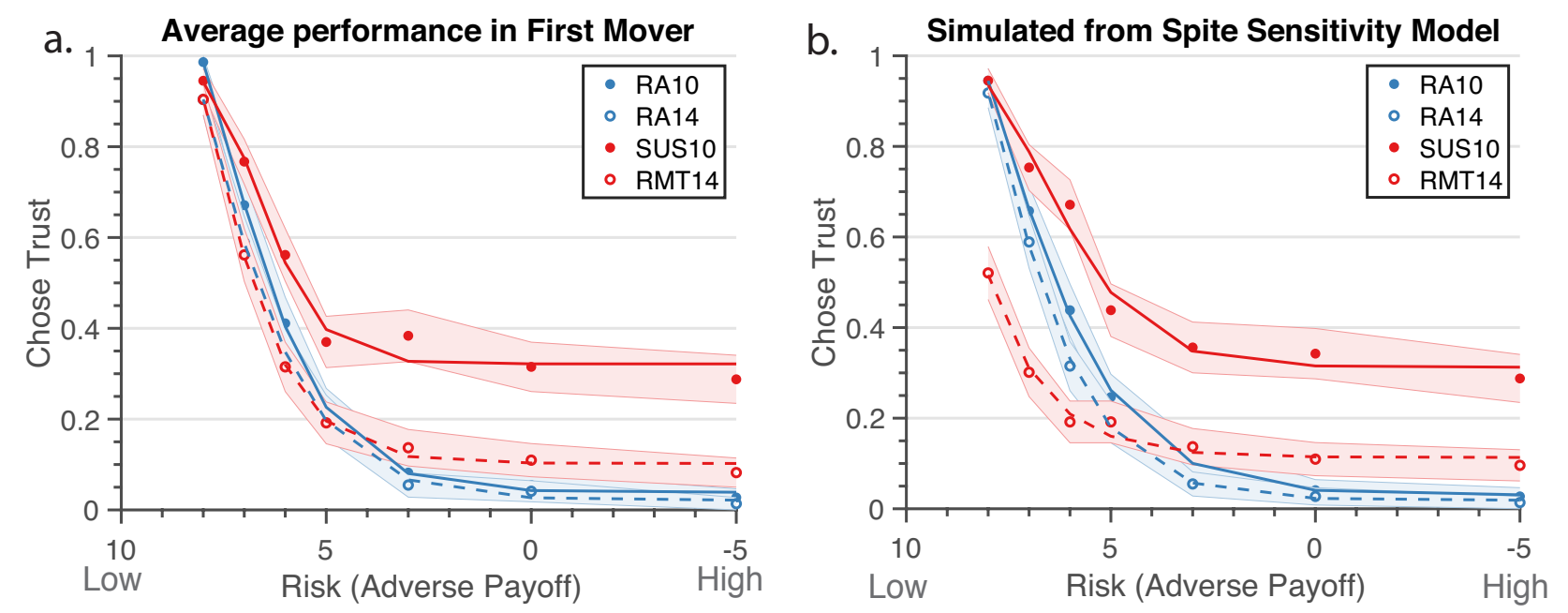

Figure 3. Replication of model comparisons. a) Average performance seen in Sample 2, in which participants in the Suspiciousness condition showed higher trust than the other conditions. $b$ ) While our model separates out the two partner conditions and in general replicated the original data well, it underestimated actual trust in the Rational Mistrust condition. Note, risk increases along the X-axis, as indicated by the decreasing Adverse Payoff. RA10 is the low temptation condition against the coin. RA14 is the high temptation condition with the coin. SUS10 is the Suspiciousness condition with the human partner. RMT14 is the Rational Mistrust condition with the human partner. The shading indicates $95 \% \mathrm{Cl}$. 
MODELING PERSECUTORY IDEATION

Model parameters and individual behavior

For Sample 1, we further assessed individual differences by fitting a Heaviside threshold for each individual over each condition as a function of the adverse payoff value, which would allow for an approximation of the level of trust in each condition (see Figure 4a for sample participant). We successfully fit Heaviside thresholds to the individual data, where the fit predicted more than $94 \%$ of the decisions made on average. Thresholds that were lower indicated more willingness to incur risk, i.e., greater trust. Like previous findings, we found a high correlation between the Risk Aversion condition thresholds associated with both the coin partner conditions, suggesting equal concern with risk irrespective of temptation $(r(241)=.784$, $p<.001$; Figure $4 \mathrm{~b}$ ), with the majority of thresholds at $\mathrm{Ad}=\$ 10$ (i.e. equal to the safe amount S). There was also a lower correlation between the two partner conditions, in which the majority of thresholds of the Rational Mistrust condition were at $\$ 10(S)$, but thresholds of the Suspiciousness condition were mostly at $A d=-\$ 5(r(241)=.175, p=.006)$. This lower correlation suggests that individuals treated the two conditions differently and that they are, in general, more willing to trust the partner when the partner is a human in the Suspiciousness condition. 

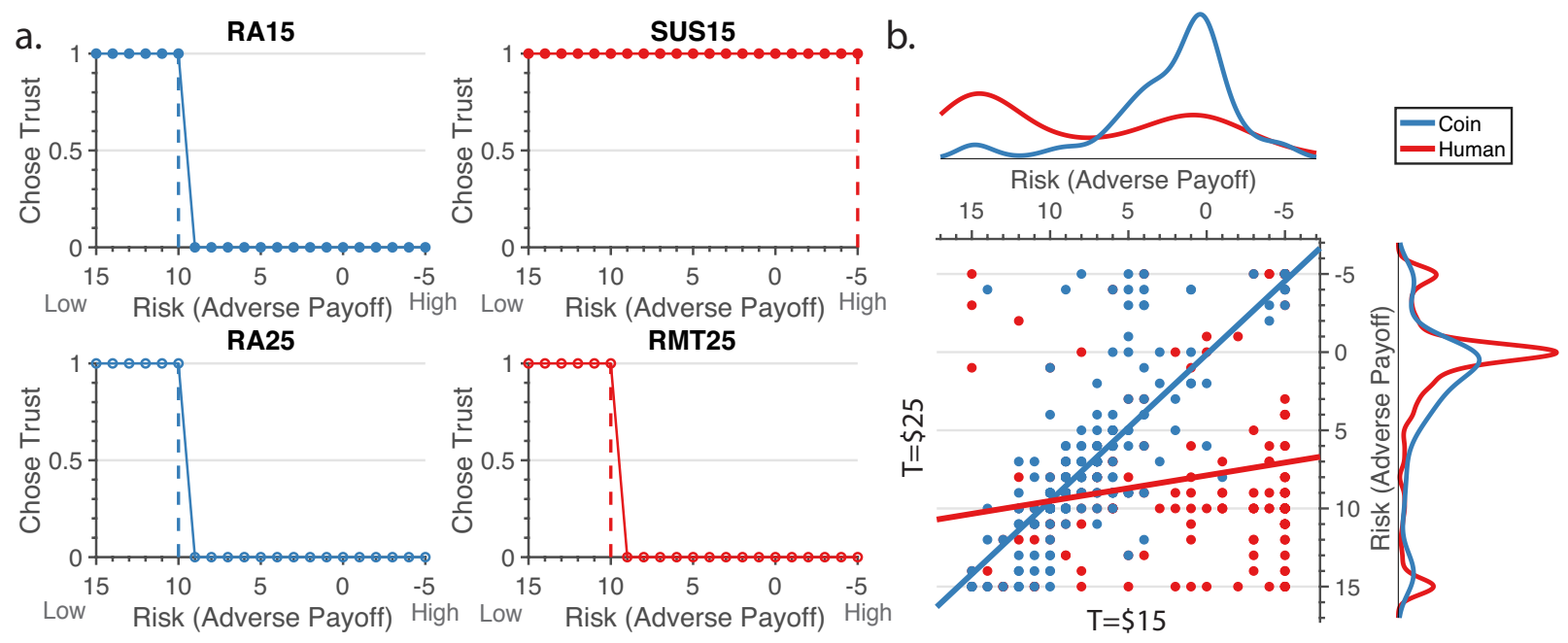

Figure 4. Individual differences in behavior. a. Example individual results for behavior, showing Heaviside threshold (dotted vertical line) determined for condition. The $y$-axis is a binary choice between trusting (1) and not trusting (0). The $x$-axis shows the adverse payoff in reverse order, such that lower thresholds (further to the right) indicate increased risk. b. Scatter plot of thresholds comparing across the low (top) and high (right) temptation $T$ values for coin conditions (blue) and partner conditions (red) in scatter plot. Above each axis, histograms show the relative count for each condition.

To further assess how well the Spite Sensitivity model represented model-agnostic measures of behavior, we examined behavioral differences by comparing the thresholds with the estimated model parameters. The estimated spite-guilt parameter in Sample 1 most negatively correlated with the Suspiciousness condition thresholds $(r(241)=-.38, p<.001$; all correlations are Spearman). It also correlated negatively with the Rational Mistrust condition thresholds $(r(241)=-.19, p=.003)$, but this correlation was smaller in magnitude (correlations significantly different, Meng's $Z=2.38, p=.009)$. A negative correlation matched our expectations that as the estimated spite-guilt parameter decreases (suggesting more spite), the thresholds with the human partner would increase (suggesting less trust). Further, we expected 


\section{MODELING PERSECUTORY IDEATION}

the Suspiciousness condition to be most associated with the estimated spite-guilt parameter. Importantly, as participants do not receive any feedback during the game, this suggests that while some individuals believe their partner to be trustworthy, others do not trust the partner even in the Suspiciousness condition, where there is no evidence that they are untrustworthy. The estimated spite-guilt parameter was not correlated with either Risk Aversion condition thresholds, which we anticipated as the estimated spite-guilt parameter was only applied to the human partner conditions.

The social risk aversion parameter was positively associated with the Rational Mistrust condition thresholds $(r(241)=.25, p<.001)$; however, it was negatively correlated with the Suspiciousness condition thresholds (SUS15: $r(241)=-.19, p=.004)$. This would suggest that in the Rational Mistrust condition, social risk aversion would increase as the risk of losing money increased, yet in the Suspiciousness condition social risk aversion would decrease as the risk increased. We did not anticipate this kind of relationship between social risk aversion and the Suspiciousness condition. Again, while there was a significant correlation between the social risk aversion parameter and the Suspiciousness condition, the correlation with the estimated spite-guilt parameter was higher (Meng's Z = 5.26, $p<.001$ ).

To further examine this relationship between social risk aversion and the human partner thresholds, we compared the difference between the two conditions, RMT25-SUS15, and identified a negative relationship $(r(241)=.288, p<.001)$. This relationship means that when RMT25 thresholds are higher than SUS15, social risk aversion is also higher such that more risk aversion in the Rational Mistrust condition drove the social risk aversion parameter estimation. 


\section{MODELING PERSECUTORY IDEATION}

As we had hoped it would represent general mistrust of other partners, it appears to match our expectations; however, the negative relationship with SUS15 thresholds seems to be driven by a disconnect between the SUS15 condition and the RMT25 condition. There was also a moderately strong correlation between the estimated spite-guilt parameter and the social risk aversion parameter $(r(241)=.490, p<.001)$. Social risk aversion was not correlated with the lower Risk Aversion condition thresholds (RA15: $r(241)=.10, p=.139$ ) and somewhat associated with the higher Risk Aversion condition thresholds (RA25: $r(241)=.13, p=.046$ ). As the social risk aversion parameter was added to the general risk aversion parameter in the human partner conditions, it is necessarily more associated with the human partner conditions.

The general risk aversion parameter was highly correlated with all conditions' thresholds, especially the coin conditions. However, it should be noted that the shape of the general risk aversion parameter was nonlinear. All other correlations comparing behavior and parameter estimates can be found in Table 1, and selected correlations can be found in the supplemental materials (Figure S2). Correlations between the parameters for Sample 1 are shown in Figure S3.

\section{Table 1}

\begin{tabular}{c|cccc}
\multicolumn{1}{c}{ PARAMETER } & RA-LOW & RA-HIGH & SUS-LOW & RMT-HIGH \\
\hline & \multicolumn{4}{c}{ Sample 1 } \\
\hline $\begin{array}{c}\text { INVERSE } \\
\text { TEMPERATURE } \\
\text { ENVY }\end{array}$ &.$- .26^{* * *}$ & $-.23^{* * *}$ & $-.24 * * *$ & -.11 \\
$\begin{array}{c}\text { ESTIMATED SPITE- } \\
\text { GUILT }\end{array}$ & $.38^{* * *}$ & $.52 * * *$ & $.15^{*}$ & $.53^{* * *}$ \\
& .02 & .01 & $-.38^{* * *}$ & $-.19 * *$
\end{tabular}




\begin{tabular}{|c|c|c|c|c|}
\hline $\begin{array}{l}\text { GENERAL RISK } \\
\text { AVERSION } \\
\text { SOCIAL RISK } \\
\text { AVERSION }\end{array}$ & $.70 * * *$ & $\begin{array}{c}.65^{* * *} \\
.13^{*}\end{array}$ & $\begin{array}{l}.34 * * * \\
-.19 * * \\
2\end{array}$ & $\begin{array}{l}.32^{* * *} \\
.25^{* * *}\end{array}$ \\
\hline $\begin{array}{c}\text { INVERSE } \\
\text { TEMPERATURE }\end{array}$ & .06 & -.13 & .17 & .11 \\
\hline ENVY & -.11 & -.15 & $-.36^{* *}$ & -.03 \\
\hline $\begin{array}{c}\text { ESTIMATED SPITE- } \\
\text { GUILT }\end{array}$ & -.11 & -.05 & $-.53 * * *$ & -.03 \\
\hline $\begin{array}{c}\text { GENERAL RISK } \\
\text { AVERSION }\end{array}$ & $.77^{* * *}$ & $.72 * * *$ & $.55^{* * *}$ & $.43 * * *$ \\
\hline $\begin{array}{l}\text { SOCIAL RISK } \\
\text { AVERSION }\end{array}$ & -.13 & -.15 & -.15 & $.37 * *$ \\
\hline
\end{tabular}

Note. Spearman correlations for each parameter (rows) by condition (columns). RA-LOW is the Risk Aversion condition against the coin partner when temptation $T$ was below the mutual payoff M; similarly, RA-HIGH refers to the Risk Aversion condition against the coin partner when temptation T was higher than the mutual payoff M. SUS-LOW refers to the Suspiciousness condition when temptation was below the mutual payoff for the human partner. RMT-HIGH refers to the Rational Mistrust condition when temptation was higher than the mutual payoff for the human partner. The top half of the table shows results for Sample 1, and the bottom half is for Sample 2. Italicized values in represent results replicated in Sample 2. $p<.05^{*}, p<.01^{* *}, p<.001^{* * *}$

We identified a computational model that was able to replicate behavioral results in the MTG. In Sample 1, estimated spite-guilt was most strongly negatively associated with thresholds in the Suspiciousness condition, which has been associated with spite sensitivity behavior previously (8). In Sample 2, we again found that the estimated spite-guilt parameter was most strongly negatively correlated with the thresholds in the Suspiciousness condition $(r$ $(71)=-.53, p<.001)$, while it was not correlated with any other conditions, which we 


\section{MODELING PERSECUTORY IDEATION}

hypothesized. Like Sample 1, the estimated spite-guilt parameter was significantly more correlated with the Suspiciousness condition than the Rational Mistrust condition (Meng's Z = $6.88, p<.001)$. When we look at the risk aversion parameters, we found that the general risk aversion was highly correlated with all conditions, as we saw in Sample 1. Finally, social risk aversion was only correlated with the Rational Mistrust condition thresholds $(r(71)=.37, p=$ .002), which was different than Sample 1 yet closer to our expectations. Like Sample 1, the social risk aversion parameter was more strongly correlated with the Rational Mistrust condition (Meng's $Z=6.84, p<.001$ ). Overall, these results validate our expectations that while both the estimated spite-guilt and the social risk aversion parameters only relate to the human partner, estimated spite-guilt is more strongly associated with the Suspiciousness condition, while social risk aversion is more associated with the Rational Mistrust condition. Again, there was a strong correlation between the social risk aversion parameter and the estimated spiteguilt parameter $(r(71)=.473, p<.001)$. Overall, these results from Sample 2 replicate findings seen in Sample 1. All correlations comparing behavior and parameter estimates can be found in Table 1. Correlations between the parameters from Sample 2 can be found in the supplemental materials (Figure S4).

\section{Model parameters and personality measures}

We additionally sought to compare individual differences on behavior and personality measures, namely the Multiphasic Personality Questionnaire (19) subscales Alienation and Harm Avoidance, to the estimated parameters. We assessed the relationship of MPQ- 


\section{MODELING PERSECUTORY IDEATION}

Alienation, associated with persecutory ideations, to MTG behavior in Sample 1 to replicate past findings that high Alienation was associated with lower trust in the Suspiciousness condition (8). We created median-split groups into high or low Alienation scores, and then conducted a repeated measures logistic regression (temptation $\mathrm{x}$ decision agent $\mathrm{x}$ adverse payoff $\mathrm{x}$ Alienation groups). We found the predicted four-way interaction effect (temptation $\mathrm{x}$ decision agent $\mathrm{x}$ adverse payoff $\mathrm{x}$ Alienation groups; $\beta=-.001, \mathrm{SE}=.003, t=-2.93, p=.003$;

Figure 5). Furthermore, there was an interaction of temptation $x$ decision agent $x$ Alienation ( $\beta$ $=.013, \mathrm{SE}=.003, t=4.65, p<.001)$. That is, when comparing the high and low Alienation groups there was a difference in thresholds that depended on the temptation and decision agent, such that those with higher Alienation were less trusting in the Suspiciousness condition but there was no difference in the other conditions. There was also an interaction of Alienation and decision agent $(\beta=-.301, \mathrm{SE}=.055, t=-5.44, p<.001)$, which showed that those with higher Alienation were less trusting with human partners, but there was no difference between the two groups in terms of coin partners. There was no main effect of Alienation $(\beta=-.015, \mathrm{SE}=$ $.045, t=-.324, p=.746)$, nor was there an interaction of Alienation with temptation $(\beta=.0012$, $\mathrm{SE}=.002, t=.647, p=.518)$ or Alienation with adverse payoff $(\beta=.004, \mathrm{SE}=.005, t=.780, p=$ .436). This result suggests a meaningful relationship between behavior in the Suspiciousness condition and Alienation, such that those with higher Alienation are more likely to be less trusting specifically when dealing with the human partner in the Suspiciousness condition, as we have seen in previous studies. 


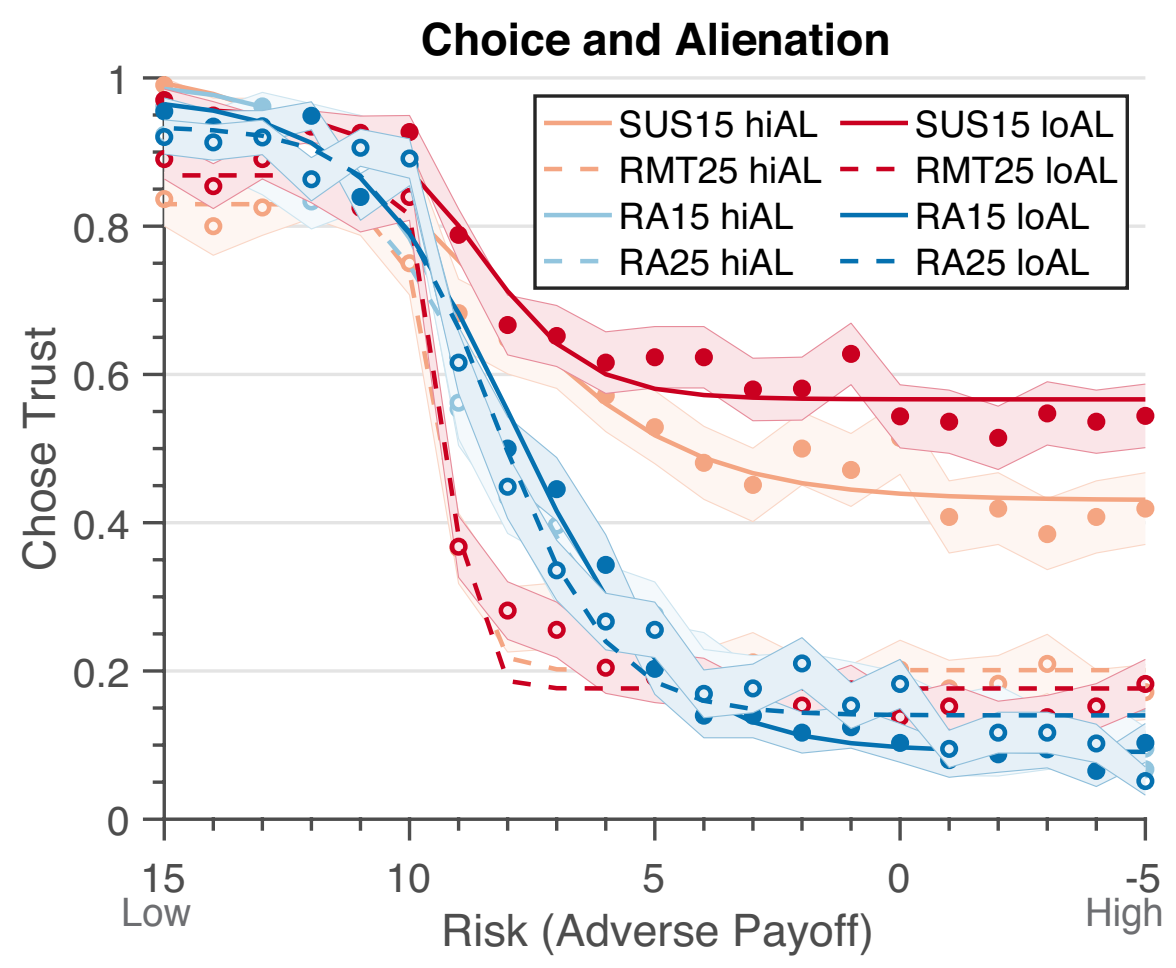

Figure 5. Sample 1 Behavior on First Mover game comparing Median Split of Alienation Scores. When comparing behavior in the first mover game between those with low (loAL) or high Alienation scores (hiAL), we found that those with higher Alienation scores are less trusting in the Suspiciousness condition (SUS15 IOAL vs. SUS15 hiAL). There were not significant differences in between the other conditions when comparing high or low Alienation. Light and dark red lines are for conditions against the human partner, while light and dark blue lines represent conditions against the coin partner. Solid lines represent low temptation trials $(T=15)$, while dashed lines represent high temptation trials $(T=25)$. Light-colored lines represent high Alienation, while dark-colored lines represent low Alienation trials.

We further examined the potential association between parameter estimates and personality measures from the MPQ. We were most interested in the Alienation subscale, which we anticipated would be related to estimated spite-guilt, and Harm Avoidance, which we anticipated would be associated with risk aversion. However, in Sample 1 we did not find a significant relationship between the general risk aversion parameter and MPQ-Harm Avoidance 


\section{MODELING PERSECUTORY IDEATION}

$(r(241)=.09, p=.090)$, nor estimated spite-guilt with MPQ-Alienation $(r(241)=-.05, p=.241)$.

In Sample 2, there was also no correlation between general risk aversion and MPQ-Harm Avoidance $(r(71)=.06, p=.315)$. We found in Sample 2 a correlation between estimated spiteguilt and MPQ-Alienation $(r(71)=-.21, p=.037)$. However, these results suggest there may be a small connection between the estimate spite-guilt parameter and a self-report measure of suspiciousness.

\section{Discussion}

The aim of this study was to create a computational model that accurately represented the patterns of behavior seen in the Minnesota Trust Game and provided clinically relevant parameters to represent persecutory beliefs. We created the model using a large sample of undergraduate students and then tested it on a second sample (8). This study was the first attempt to build an econometric model of the unique circumstances examined in the Minnesota Trust Game. We developed and replicated in a separate sample a model based on the Fehr-Schmidt Inequity Aversion model that not only calculated players' envy, but also their estimation of the second mover's spite as an expansion of guilt into negative values. Our model performed better than both a random model and the original Fehr-Schmidt model (20), which could not explain spite sensitivity in the MTG. The final model also included two risk aversion parameters, relating to overall risks and risks specific to the human interactions. The estimated spite-guilt parameter was most related to suspicious behavior in the task, suggesting that it captures spite sensitivity, whereas the general risk aversion was strongly associated with less 


\section{MODELING PERSECUTORY IDEATION}

trust in the game in all conditions. Social risk aversion, in contrast, was most strongly associated with Rational Mistrust. These results suggest that fear of spite and social risk aversion are separable mechanisms in decision-making. Furthermore, Sample 1 replicated a significant relationship between MPQ-Alienation, a personality measure of persecution, and the Suspiciousness condition in the Minnesota Trust Game that specifically targets spite sensitivity.

In past studies of the Trust Game, participants tend to send less money when they know their partner is a computer (29), suggesting they apply different expectations depending on the decision agent. In a similar two-person social decision making game, the Ultimatum Game, rejection of unfair options are more common for human than computer partners due to the expectation that humans, but not computers, would understand that it was unfair (30). In the Minnesota Trust Game, past research has also found distinctions in behavior between the coin and human partners (8). Taken together, these results show that the presumed intentions and mental capacity of a partner are critical to the social interaction. Our final model identified two instances in which treating the decision agents (coin and human) separately improved the model. Estimated spite-guilt (modeled as a prediction of the partner's spite or guilt along a continuum) was only applied to the human partner. This additional parameter assumes some "mentalizing" on the part of the partner, but not coin; additional attempts to model mentalizing in the coin were not effective (see supplemental materials). Additionally, we had two separate risk aversion parameters for overall risk and risk associated with only the human partner interactions. While it is uncommon to separate risk aversion across two decision agents, it speaks to separate approaches to the two decision agents in our game. Due to the nature of 


\section{MODELING PERSECUTORY IDEATION}

our paradigm, treating the decision agents separately allowed us to measure distinct beliefs about the partners' intentions.

Risk aversion is a common parameter used to assess the dislike of gambling or uncertainty in losing money $(26,31,32)$. Past research points towards a difference between risk aversion and a lack of trust in the Trust Game $(33,34)$. Computational modeling of the multiround Trust Game has shown that risk aversion (the value of money kept over potential money gained), played an important role comparing the potential loss regardless of the partner's cooperativity (26). Because we manipulated risk as a parametric variable, we anticipated that risk aversion would play an important role in decision making in this game yet would not fully explain behavior in the Suspiciousness condition. Our best model included risk aversion parameters with two different functions, as mentioned above. This social risk aversion parameter was also most associated with the Rational Mistrust condition, showing its ties to human mistrust. Against expectations, we did not find a significant relationship between MPQHarm Avoidance and general risk aversion (8), perhaps because those questions focus on physical rather than financial risks. Overall, the inclusion of risk aversion parameters is valuable in contextualizing the fear of losing money versus partner distrust.

We found that the additional estimated spite-guilt parameter was important to model suspicious behavior, thus allowing us to model spite sensitivity for the first time (H4a). In contrast, separating positive and negative guilt into guilt and spite did not improve the model (H4b). Uniquely, this model extended the estimated spite-guilt parameter to include negative values. The original Fehr-Schmidt model did not allow for negative parameters in order to test 


\section{MODELING PERSECUTORY IDEATION}

specific individuals who deviated from equilibrium, and it is uncommon in other uses of this model (35). However, as we are interested in an individual's perception of spiteful partners, including a negative range for estimated spite-guilt allowed us to do this parsimoniously as a continuous variable. We found one article that referred to spite and guilt as opposite responses in a public goods game (36). Guilt is thought to be an important driver in enhancing relationships and social norms (37). Modeling of the second mover's perspective therefore focuses on guilt aversion, which encompasses both maximizing outcomes and minimizing anticipated guilt from letting down a partner (38). However, guilt aversion is similarly only allowed to be positive, and testing the propensity for spite is less common in trust games; thus, we are limited in our ability to examine if spite and guilt are truly a continuum. However, our results suggest it is an effective way to model fear of spite in the Minnesota Trust Game. Importantly, as predicted this parameter was most associated with the Suspiciousness condition in both samples. The estimated spite-guilt parameter was key to understanding spite sensitive beliefs about a partner, above-and-beyond risk aversion.

While estimated spite-guilt captures the participant's beliefs about a partner's intentions, how well does it capture theory of mind? Several theories exist around the role of theory of mind in persecutory ideation. As persecutory ideations are unfounded beliefs that another has ill intentions towards oneself, one theory is that they are driven by the inability to understand or intuit another's intentions. Theory of mind, in its most basic form, refers to the capacity to represent one's own and other persons' mental states (28). Key to this idea is that other's mental states may also be different from our own, and that contextual cues like 


\section{MODELING PERSECUTORY IDEATION}

behavioral gestures, facial expressions of emotions, and situational events can provide important information to understand different views. Broadly, substantial theory of mind deficits have been observed in individuals with first episode psychosis, ultra-high risk for psychosis, and unaffected relatives (39). Additional research suggests that these findings persist into chronic psychosis (40).

However, the specificity of the role of theory of mind in persecutory delusions is less certain. Recent work has instead suggested that hyper-mentalization (i.e. over-attribution of intentions to random movements) in patients was associated with positive symptoms like delusions, namely suspiciousness, thus showing that persecutory beliefs may be related to poor theory of mind due to over - not under -- use (41). Additionally, there is evidence that deficits in mental state reasoning, defined as the ability to infer others' motives and intentions, moderate the relationship of persecutory ideation and social functioning. One study identified a negative relationship between persecutory ideation and social functioning when mental state reasoning was in the bottom $78^{\text {th }}$ percentile, but there was no relationship when mental state reasoning was very high. In contrast, mental state decoding, defined as the ability to infer emotions and feelings from others, was not a significant moderator (42). Other studies have not found a strong relationship between theory of mind and persecutory ideation. For example, perspective-taking behavior was not associated with paranoia in a group of patients with schizophrenia (43). A recent meta-analysis of delusions suggested that the theory of mind account does not have strong support, instead finding theory of mind is more associated with negative symptoms (44) like flat affect, anhedonia, and apathy (45). For the current study, we 


\section{MODELING PERSECUTORY IDEATION}

did not anticipate theory of mind deficits in our sample and did not assess them. In its most basic form, we can identify that participants believe that the human partner has distinct intentions from the coin, as they treated the two decision agents differently. When it comes to more complex theory of mind reasoning, it becomes more difficult to assess its role in decision making in the MTG. For example, to understand the incentives for the partner in the Suspiciousness condition, one must understand that the partner would likely want to maximize their outcome. A failure to understand this motivation for the partner may also look like distrust of the partner. Therefore, theory of mind may be an additional factor involved in this task that we cannot directly address in the current study yet may not play an important role in the association with persecutory beliefs.

There remain open questions for our model. There was some overlap in the relationships of the estimated spite-guilt and social risk aversion parameters. This included having both correlate with both the Suspiciousness and Rational Mistrust conditions, albeit with stronger relationships with the expected conditions. Additionally, social risk aversion and estimated spite-guilt parameters are correlated themselves. As both parameters were included in only the human partner trials, it is reasonable that both would be associated with the two human partner trials. Importantly, we were still able to recover the parameters with good replication of the expected outcomes in behavior despite these overlapping parameters. However, we did not predict a negative relationship between social risk aversion and the Suspiciousness condition thresholds. Additionally, we found that when there is a larger discrepancy between the two parameters, social risk aversion accounted for the lower trust in 


\section{MODELING PERSECUTORY IDEATION}

the Rational Mistrust condition and therefore had a negative correlation with the greater trust in the Suspiciousness condition. These findings suggest that social risk aversion and estimated spite-guilt parameters interact. One explanation for this relationship is that those who are specifically suspicious of their partner's intent would also be more concerned about risk taking with a human partner in any situation. However, the estimated spite-guilt parameter is most strongly associated with the Suspiciousness condition in both samples.

We also found mixed results about the relationship between estimated spite-guilt and the MPQ Alienation scale, which we had hypothesized would be related. Both behavioral samples found that higher Alienation was associated with less trust in the Suspiciousness condition behavior when we separated the sample into high and low Alienation groups. However, there was only a significant correlation between Alienation and estimated spite-guilt in Sample 2. One explanation for the difference across samples may be that the two games varied in the values used for the game. It is also possible that the positive correlation between the self-reported measure of alienation and the estimated spite-guilt parameter was due to chance, as the sample size is lower on Sample 2. Alternatively, a potentially important difference between the samples is that we paid participants in the Sample 2, but not Sample 1, which may mean individuals were more incentivized to reveal their true preferences in Sample

2. There is mixed evidence that incentive payments influence decisions to trust differently than hypothetical payments. Previous research has shown a minimal difference in behavior between hypothetical and incentivized outcomes in the Trust Game (46). In a similar case, trust outcomes were similar when comparing monetary and non-monetary incentives (47). However, 


\section{MODELING PERSECUTORY IDEATION}

the opposite has also been observed. A meta-analysis found that when making similar comparisons of real payments versus randomized lottery payments (i.e. randomly selecting an individual to actually pay them by their choice), individuals sent less money when it was a randomized choice (29). Similarly, Holm \& Nystedt (2008) found that those given hypothetical outcomes were much less willing to trust than those with real payments or random lottery payments (48). In comparison with our results, it may be the case that real payment led to a difference in behavior in the task in Sample 2 compared to Sample 1, although it is difficult to assess with the current data. Finally, we also used undergraduate students for both samples, who may feel less persecuted than the broader community, thus reducing variability. Future studies should disentangle between these different possibilities.

Our model of the Minnesota Trust Game is the first to distinguish behavior related to spite sensitivity that predicts behavior in the Suspiciousness condition, which assesses irrational fear of a partner's likelihood to be spiteful. As spite is hypothesized to represent a behavior that is evolutionarily adaptive for kin selection (1), spite sensitivity may represent a prior expectation that out-group individuals will exclude or persecute an individual despite no direct benefit to the persecutor. This model is a first step to measuring persecutory ideation in a healthy sample in hopes of further elucidating mechanisms of persecutory ideation. Our findings support hypotheses that prior beliefs about a partner's intentions influence decisions to trust. It will be useful to compare behavior in the task with individuals who have experienced persecutory ideation, and if successful to use it as a tool to examine the representation of spite sensitivity in the brain. Finally, modeling the Minnesota Trust Game could prove useful for 


\section{MODELING PERSECUTORY IDEATION}

examining treatments that target persecutory beliefs - for example, by allowing observations of an effect of these interventions on the estimated spite-guilt parameter. 


\section{Methods}

\section{Participants}

All subjects provided informed consent through the University of Minnesota Internal Review Board. Sample 1 consisted of 251 undergraduate psychology students (age mean =19.9 (3.4), range $18-46$ years; $62 \%$ female; $12.9(S D=1.3$ ) years of education) who completed the Minnesota Trust Game (MTG) and a series of personality questionnaires in individual lab sessions. Participants received course extra-credit for their participation and all game payments were imaginary. Eight subjects were excluded for incomplete data on the task. We also analyzed a second sample of data that was previously reported (8). Sample 2 consisted of 82 undergraduate psychology students who were tested in batches of 4-14. Sample 2 participants received extra-credit for participating in the experiment and were also paid cash based on two randomly selected trials with random pairings with other study participants. Nine subjects were excluded due to poor task comprehension and a pattern of inconsistent responding (8).

\section{Questionnaires}

The personality inventory included items from the Multidimensional Personality Questionnaire Brief Form (MPQ-BF) (19). MPQ-BF subscales included the Alienation subscale. The Alienation subscale quantifies suspiciousness in day-to-day life and contains items such as "some people are against me for no good reason." Items were randomized throughout the questionnaire and scored on a 4-point Likert scale $(1=$ always true, $2=$ mostly true, $3=$ mostly 


\section{MODELING PERSECUTORY IDEATION}

false, 4 = always false). T-scores were calculated for the MPQ-BF as per Patrick and colleagues (2002) using software available at dionysus.psych.wisc.edu/arl/downloads.html.

\section{Minnesota Trust Game}

The Minnesota Trust Game (8) is a computerized, parametric, non-iterative economic decision-making task comprised of two sub-games, the First Mover and Second Mover Games. We asked individuals to play against a decision-agent; two conditions were used in which they played against another participant in the research study or a fair "coin" which made 50/50 decisions. The experimenter emphasized that the decisions the participant made would determine their own and another player's winnings in a randomly selected trial. In the case of a coin, the participant would flip the coin to determine the outcome for that trial.

Participants played both the First and Second Mover Games, and they played the Second Mover Game first. Our modeling focused only on the First Mover Game. For the First Mover Game ( $1^{\text {st }} \mathrm{M}$ Game), they made the first decision in a two-player turn-based game. Here the participant decided whether to accept a smaller, safe reward $S$ ( $\$ 10$ for Sample 1$)$ or have their partner decide the outcome and potentially increase their mutual reward for that trial $(M$; $\$ 20$ for Sample 1). As shown in the top node of the decision-tree (Figure 1), while playing the role of the first mover the participant chose between the assured payoff $S$ and the alternative payoff (to trust, or take a chance on, the second mover). Participants in the $1^{\text {st }} \mathrm{M}$ Game were told whether the decision-agent was another participant in the study or a coinflip representing a passive partner. The alternative payoff consisted of two conditions, Rational Mistrust and 


\section{MODELING PERSECUTORY IDEATION}

Suspiciousness, which was distinguished by the value of the second mover's potential winnings, the temptation $T$. The range of the adverse payoff $A d$ is described in Figure 1. No-risk trials ( $A d$ values above S) provided a validity probe to determine whether participants understood the experimental manipulations and allowed us to examine risk tolerance across a spectrum of choices.

The Rational Mistrust condition was the first mover's decision to trust or not when the second mover had a monetary incentive to choose the temptation over the mutual reward $(T=$ $\$ 25$ in Sample 1), leaving the first mover with the adverse payoff $A d$. The Suspiciousness condition was set up so that the second mover had a monetary disincentive to select the temptation $T$ (\$15 in Sample 1). When the decision agent was a random coin, the Risk Aversion conditions paralleled the Rational Mistrust and Suspiciousness conditions, but they differed in that the first mover chose between the assured payoff and allowing the coin to determine their winnings. Thus, choosing the assured payoff in this game indicated a simple aversion to that risk. In the $1^{\text {st } M}$ Game, participants did not receive any feedback from their partners. Sample 2 had lower values for all the variables: the assured payoff $S$ was $\$ 8$ and the mutual reward $M$ was $\$ 12$. The Rational Mistrust condition $T$ temptation was $\$ 14$, while the Suspiciousness condition $T$ temptation was $\$ 10$. The adverse payoff $A d$ values ranged across a spectrum of values (see Figure 1). The Second Mover Game ( $2^{\text {nd }} M$ Game) had the same contingencies but was from the perspective of the second mover. 


\section{MODELING PERSECUTORY IDEATION}

\section{Design}

For Sample 1, each participant made 42 unique decisions in the simpler, $2^{\text {nd }} \mathrm{M}$ Game (21 decisions in each condition, to test adverse payoffs for each value $-\$ 5$ to $\$ 15)$ and then made 84 unique decisions in the $1^{\text {st }} \mathrm{M}$ Game (21 in each condition for both partner and coin decision agents). The $2^{\text {nd }} \mathrm{M}$ Game was performed before the $1^{\text {st }} M$ Game, to ensure that participants understood what their partner had been told when making decisions. For Sample 2, each participant made 14 decisions in the $2^{\text {nd }} \mathrm{M}$ Game, and then made the same decisions in the $1^{\text {st }} \mathrm{M}$ Game with the two decision agents (coin and human partner), summing to 28 choices. Choices were presented in a random order. The range of the adverse payoff varied in a parallel manner within each condition of the First and Second Mover games, resulting in a range of financial risk levels within each condition and a parametric repeated measures experimental design. For each choice participants were shown the fixed values of the assured payoff and mutual reward alongside changing elements which were the type of decision agent (partner or coin), the temptation, and the adverse payoff. There was no feedback regarding the outcome of participants' decisions until the end of the experiment. That is, each trial was treated as a single interaction. Furthermore, there was no deception.

\section{Analysis}

The outcome variable was the participant's choices during the MTG. In the $1^{\text {st } M}$ Game, the choices were coded ' 0 ' if the participant chose the assured payoff and ' 1 ' if the participant ceded the choice to the decision-agent. Our analyses focus only on the $1^{\text {st }} \mathrm{M}$ Game responses. 


\section{MODELING PERSECUTORY IDEATION}

We ran a repeated measures logistic regression with Condition (Rational Mistrust or Suspiciousness) by Alienation score (High or Low) by Adverse Payoff (each value offered) by Decision Agent (coin or human). Furthermore, in order to get a discrete measure for each individual, we leveraged the parametric manipulation of the adverse payoff to determine each participant's change point from ceding the choice (or trusting) to the assured payoff (or not trusting) by fitting their choices using a Heaviside function, which relies on maximum likelihood estimation. We conducted Spearman correlations between the parameter estimations and thresholds for each condition. Finally, we conducted one-tailed Spearman correlations between the estimated spite-guilt parameter and MPQ-Alienation, which we predicted would be negatively correlated. We also conducted one-tailed Spearman correlations between the General Risk Aversion parameter and MPQ-Harm Avoidance, which we anticipated would be positively correlated. Both of these hypotheses were predicted from previous research with the MTG (8).

\section{Modeling}

The aim of this analysis was to create a model that provided behavioral realism, i.e., a model that accurately described the behavioral phenomenon seen in the MTG, where individuals choose not to trust in an instance where it might be reasonable to do so (Suspiciousness condition). Additionally, we intended for the model to provide interpretable results when comparing individual differences in behavior. A final goal was that the model would identify individual differences in paranoid beliefs that could be quantified by personality 


\section{MODELING PERSECUTORY IDEATION}

measurements. Therefore, we focus parameters on representing behaviors related to suspiciousness.

We developed normative models in MATLAB (49) based on the Fehr \& Schmidt (1999) inequity aversion model (50) of two-player trust games to calculate utility $(U)$ of a choice:

$$
U_{1}(x)=x_{1}-\alpha_{1} \max \left\{x_{2}-x_{1}, 0\right\}-\beta_{1} \max \left\{x_{1}-x_{2}, 0\right\} \quad \text { Eq. } 1
$$

where $\mathrm{x}$ is defined as the offer, and the subscripts are simplified to players $1 \& 2$ (first mover and second mover, respectively). This included two main parameters: envy $\left(\alpha_{1}\right)$ and guilt $\left(\beta_{1}\right)$. Envy described the participant's dislike of potential unfairness when their partner receives a larger payment. Guilt described the participant's dislike of potential unfairness (hence in terms of unequal distribution of payoffs) when offered more money than their partner.

We tested several different parameters (always using Sample 1), optimizing model fit according to Bayesian Information Criteria (BIC) (51). The benefit of BIC is that it corrects for a higher number of parameters. Parameters were inferred by the model by fitting the choices using maximum likelihood estimation. The parameters from the model were tested for the extent to which they represented spite sensitivity, a phenomenon whereby players distrust a partner despite the partner's disincentive to betray the player. For the purposes of understanding the direction of the parameter, we will refer to it as the estimated spite-guilt parameter, as low values represent spite sensitivity, while high values represent guilt. To test individual differences, each parameter was estimated for each individual participant. 


\section{MODELING PERSECUTORY IDEATION}

Based on the Fehr-Schmidt model, the utility of the assured payoff $U_{\text {ASSURED }}$ was always $S$, because there was no inequality between the two players, and the value was constant. Similarly, the utility of the mutual payoff $U_{\text {MUtUAL }}$ was always $M$. Our main target for modeling lay in modeling the utility of the adverse payoff $\left(U_{A D V E R S E}\left(x_{1}\right)\right)$. In its simplest form, the adverse payoff was the value of the amount of money received by player 1 .

However, the decision is not simply among these three options, but rather between one assured option and one alternative option: to trust the partner. Therefore, we need to calculate the utility of trusting the partner $\left(U_{\text {TRUST }}\left(x_{1}\right)\right)$, which we modeled by weighting the partner's options with probability $(p)$ that the partner will choose the adverse payoff (Eq. 2). In the simplest forms of the model, $p$ is defined as .5 to denote random choice between the adverse and mutual payoffs. This connotes the Risk Aversion conditions. In more complex forms (i.e., modeling the estimation of the partner's choice), $p$ is calculated based on the estimated parameters (see H4a).

$$
U_{T R U S T}\left(x_{1}\right)=p\left(U_{A D V E R S E}\right)+(1-p)\left(U_{M U T U A L}\right) ; \text { Eq. } 2
$$

Finally, to incorporate a measure of stability in decision making in the individual, we included a parameter for inverse temperature $(\lambda)$, which represented the level of randomness in a decision. This means that the higher $\lambda$, the more consistent an individual is on their decision. We use a softmax equation to calculate the probability of a choice using $\lambda$ (Eq. 3). The probability for each choice was then compared to the choices of the individual to calculate the negative log likelihood, which was then minimized to identify the best parameter estimates. 
MODELING PERSECUTORY IDEATION

$$
\text { probability (TRUST) }=\frac{e^{\lambda * U_{T R U S T}}}{e^{\lambda * U_{S A F E}+e^{\lambda * U_{T R U S T}}}} ; \text { Eq. } 3
$$

This setup was the basic modeling procedure. Specific models for each hypothesis are laid out in the supplemental materials, and we describe the best model in the results.

\section{Stopping criteria}

The model captures the distinctions between Rational Mistrust and Suspiciousness conditions, as well as distinction between the coin and human partners. To test the efficacy of the model in capturing these distinctions, we applied the parameter estimates collected for each individual to simulate choice outputs and compared the outcome to the original choices.

\section{Data Simulation}

Data was simulated using the parameters extracted by the participants in order to attempt to recover behaviors seen in the participants using MATLAB.

\section{Data Availability Statement}

As our sample was collected before data repositories were common, our original IRB did not account for this type of data sharing. Currently we are unable to make our data publicly available. However, our code is publicly available on the Open Science Foundation

\section{https://osf.io/fhqj6/.}


MODELING PERSECUTORY IDEATION

\section{Acknowledgements}

We thank A. David Redish for his valuable feedback. 


\section{MODELING PERSECUTORY IDEATION}

\section{References}

1. Gardner A, West SA. Spite. Curr Biol. 2006;16(17):662-4.

2. West SA, Gardner A. Altruism, spite, and greenbeards. Science (80- ). 2010;327(5971):1341-4.

3. Giron D, Dunn DW, Hardy ICW, Strand MR. Aggression by polyembryonic wasp soldiers correlates with kinship but not resource competition. Nature. 2004;430(7000):676-9.

4. American Psychiatric Association. Diagnostic and statistical manual of mental disorders. 5th ed. Arlington, VA: American Psychiatric Publishing; 2013.

5. Verdoux $\mathrm{H}$, Van Os J. Psychotic symptoms in non-clinical populations and the continuum of psychosis. Schizophr Res. 2002;54(1-2):59-65.

6. Freeman D, Garety PA, Bebbington PE, Smith B, Rollinson R, Fowler D, et al. Psychological investigation of the structure of paranoia in a non-clinical population. Br J Psychiatry. 2005;186(MAY):427-35.

7. Bebbington PE, McBride O, Steel C, Kuipers E, Radovanovic M, Brugha T, et al. The structure of paranoia in the general population. Br J psychiatry [Internet]. 2013 Jun [cited 2016 Jun 9];202:419-27. Available from:

http://www.ncbi.nlm.nih.gov/pubmed/23661767

8. Johnson MK, Rustichini A, MacDonald III AW. Suspicious personality predicts behavior on a social decision-making task. Pers Individ Dif [Internet]. 2009 Jul [cited 2011 Jul 27];47(1):30-5. Available from: 


\section{MODELING PERSECUTORY IDEATION}

http://linkinghub.elsevier.com/retrieve/pii/s0191886909000543

9. Bagby RM, Rogers R. Detecting Malingered and Defensive Responding on the MMPI-2 in a Forensic Inpatient Sample. J Pers Assess. 1994;62(2):191-203.

10. Fehr E, Camerer CF. Social neuroeconomics: the neural circuitry of social preferences. Trends Cogn Sci. 2007;11(10):419-27.

11. Sanfey AG. Social Decision-Making: Insights from Game Theory and Neuroscience. Science (80- ). 2007;318:598-603.

12. Rilling JK, Sanfey AG. The Neuroscience of Social Decision-Making. Annu Rev Psychol. 2011;62:23-48.

13. Ruff CC, Fehr E. The neurobiology of rewards and values in social decision making. Nat Rev - Neurosci [Internet]. 2014;15:549-62. Available from:

http://dx.doi.org/10.1038/nrn3776

14. King-Casas B, Chiu PH. Illness Through Multiplayer Economic Games. Biol Psychiatry [Internet]. 2012;72(2):119-25. Available from:

http://dx.doi.org/10.1016/j.biopsych.2012.03.033

15. Berg J, Dickhaut J, McCabe KA. Trust, Reciprocity, and Social History. Games Econ Behav. 1995;10:122-42.

16. Applebaum PS, Robbins PC, Roth LH. Dimensional approach to delusions: Comparison across types and diagnoses. Am J Psychiatry. 1999;156:1938-43.

17. Fett A-KJ, Shergill SS, Joyce DW, Riedl A, Strobel M, Gromann PM, et al. To trust or not to trust: the dynamics of social interaction in psychosis. Brain. 2012;135:976-84. 


\section{MODELING PERSECUTORY IDEATION}

18. Fett A-KJ, Shergill SS, Korver-Nieberg N, Yakub F, Gromann PM, Krabbendam L. Learning to trust: Trust and attachment in early psychosis. Psychol Med. 2016;46(7):1437-47.

19. Patrick CJ, Curtin JJ, Tellegen A. Development and validation of a brief form of the Multidimensional Personality Questionnaire. Psychol Assess. 2002;14(2):150-63.

20. Fehr E, Schmidt KM. A theory of fairness, competition, and cooperation. Q J Econ. 1999;(August):817-68.

21. Nash J. Equilibrium points in n-person games. Proc Natl Acad Sci. 1950;36:48-9.

22. Nash J. Non-Cooperative Games. Ann Math. 1951;54(2):286-95.

23. Bolton GE, Ockenfels A. ERC: a theory of euity, reciprocity, and competition. Am Econ Rev. 2000;90:166-93.

24. Charness G, Rabin M. Understanding social preferences with simple tests. Q J Econ. 2002;117:817-769.

25. Krajbich I, Adolphs R, Tranel D, Denburg NL, Camerer CF. Economic games quantify diminished sense of guilt in patients with damage to the prefrontal cortex. J Neurosci [Internet]. 2009;29(7):2188-92. Available from:

http://www.pubmedcentral.nih.gov/articlerender.fcgi?artid=2646169\&tool=pmcentrez\& rendertype=abstract

26. Hula A, Vilares I, Lohrenz T, Dayan P, Montague PR. A model of risk and mental state shifts during social interaction. PLoS Comput Biol [Internet]. 2018;14(2):1-20. Available from: http://dx.doi.org/10.1371/journal.pcbi.1005935

27. Bolton GE, Ockenfels A. ERC: A Theory of Equity, Reciprocity, and Competition. Am Econ 


\section{MODELING PERSECUTORY IDEATION}

Rev. 2000;90(1):166-93.

28. Premack D, Woodruff G. Does the chimpanzee have a theory of mind? Behav Brain Sci. 1978;4(1978):515-26.

29. Johnson ND, Mislin AA. Trust games: A meta-analysis. J Econ Psychol [Internet]. 2011;32:865-89. Available from: http://dx.doi.org/10.1016/j.joep.2011.05.007

30. Radke S, Güroğlu B, de Bruijn ERA. There's something about a fair split: Intentionality moderates context-based fairness considerations in social decision-making. PLoS One. $2012 ; 7(2)$.

31. Levy I, Snell J, Nelson AJ, Rustichini A, Glimcher PW. Neural representation of subjective value under risk and ambiguity. J Neurophysiol. 2010;103(2):1036-47.

32. Glimcher PW. Understanding risk: A guide for the perplexed. Cogn Affect Behav Neurosci [Internet]. 2008;8(4):348-54. Available from:

http://www.springerlink.com/index/10.3758/CABN.8.4.348

33. Bohnet I, Zeckhauser R. Trust, risk and betrayal. J Econ Behav Organ. 2004;55(4 SPEC.ISS.):467-84.

34. Houser D, Schunk D, Winter J. Distinguishing trust from risk: An anatomy of the investment game. J Econ Behav Organ [Internet]. 2010;74:72-81. Available from: http://dx.doi.org/10.1016/j.jebo.2010.01.002

35. Yang Y, Onderstal S, Schram A. Inequity aversion revisited. J Econ Psychol [Internet]. 2016;54(135):1-16. Available from: http://dx.doi.org/10.1016/j.joep.2015.12.009

36. Chan KS, Godby R, Mestelman S, Muller RA. Spite, Guilt and the Voluntary Provision of 


\section{MODELING PERSECUTORY IDEATION}

Public Goods When Income Is Not Distributed Equally. Can J Econ. 1996;29:S605-9.

37. Baumeister RF, Stillwell AM, Heatherton TF. Guilt: An Interpersonal Approach. Psychol Bull. 1994;115(2):243-67.

38. Battigalli BP, Dufwenberg M. Guilt in Games. 2006;97(2).

39. Bora E, Pantelis C. Theory of mind impairments in first-episode psychosis, individuals at ultra-high risk for psychosis and in first-degree relatives of schizophrenia: Systematic review and meta-analysis. Schizophr Res [Internet]. 2013;144(1-3):31-6. Available from: http://dx.doi.org/10.1016/j.schres.2012.12.013

40. Bora E, Yucel M, Pantelis C. Theory of mind impairment in schizophrenia: Meta-analysis. Schizophr Res [Internet]. 2009;109(1-3):1-9. Available from:

http://dx.doi.org/10.1016/j.schres.2008.12.020

41. Hajdúk M, Pavelková L, Ohrablo P, Petrušová V, Keretik A, Forgáčová L. Attribution of Intentions in Patients with Schizophrenia Spectrum Disorders with Persecutory Delusions. Abstr Sixth Bienn SIRS Conf. 2018;S250.

42. Phalen PL, Dimaggio G, Popolo R, Lysaker PH. Aspects of Theory of Mind that attenuate the relationship between persecutory delusions and social functioning in schizophrenia spectrum disorders. J Behav Ther Exp Psychiatry [Internet]. 2017;56:65-70. Available from: http://dx.doi.org/10.1016/j.jbtep.2016.07.008

43. Korver-Nieberg N, Fett AKJ, Meijer CJ, Koeter MWJ, Shergill SS, De Haan L, et al. Theory of mind, insecure attachment and paranoia in adolescents with early psychosis and healthy controls. Aust N Z J Psychiatry. 2013;47(8):737-45. 


\section{MODELING PERSECUTORY IDEATION}

44. Garety PA, Freeman D. The past and future of delusions research: From the inexplicable to the treatable. Br J Psychiatry. 2013;203(5):327-33.

45. Andreasen NC. Schizophrenia: Positive and Negative Symptoms and Syndromes. Mod Trends Pharmacopsychiatry. 1990;24:73-88.

46. Thielmann I, Heck DW, Hilbig BE. Anonymity and incentives: An investigation of techniques to reduce socially desirable responding in the trust game. Judgm Decis Mak. 2016;11(5):527-36.

47. Luccasen RA, Thomas MK. Monetary incentives versus class credit: Evidence from a large classroom trust experiment. Econ Lett [Internet]. 2014;123(2):232-5. Available from: http://dx.doi.org/10.1016/j.econlet.2014.02.012

48. Holm H, Nystedt P. Trust in surveys and games - A methodological contribution on the influence of money and location. J Econ Psychol. 2008;29(4):522-42.

49. MATLAB. 9.7.0.1190202 (R2018b). Natick, Massachusetts: The MathWorks Inc.; 2018.

50. Fehr E, Schmidt KM. A theory of fairness, competition, and cooperation. Q J Econ. $1999 ; 817-68$.

51. Schwarz GE. Estimating teh dimension of a model. Ann Stat. 1978;6(2):461-4. 